\title{
Quality Analysis of Antimicrobial Restriction Policy in Pediatrics
}

\author{
Andre Ricardo Araujo da Silva ${ }^{1}$ and Deborah Cardoso Albernaz de Almeida Dias ${ }^{1,{ }^{*}}$ \\ ${ }^{1}$ Saúde Materno-Infantil Post Graduation, Faculty of Medicine, Universidade Federal Fluminense, Niterói, Brazil \\ "Corresponding author: Saúde Materno-Infantil Post Graduation, Faculty of Medicine, Universidade Federal Fluminense, Zip Code: 24033-900, Niterói, Brazil. Tel: \\ +55-2126299360, Email: deborahalbernaz@hotmail.com
}

Received 2020 January 14; Revised 2020 October 18; Accepted 2020 November 26.

\begin{abstract}
Background: Antimicrobial stewardship programs (ASPs) are coordinated programs developed in recent years to promote the appropriate use of antimicrobials and reduce microbial resistance. One important action for a successful ASP is the implementation of an antimicrobial policy restriction.

Objective: The study analyzed the quality of target-antimicrobial requisitions after the introduction of an antimicrobial policy restriction for children.

Methods: We conducted a retrospective study in a neonatal intensive care unit (NICU) and three pediatric intensive care units (PICUs). An ASP was implemented in October 2016, and 14 target antimicrobials were selected to be prescribed after pre-approval by a pediatric infectious disease specialist. All requisitions were analyzed according to indication, antimicrobial type, dose, duration, and collection of cultures before administration. There were no exclusion criteria for requisition analysis.

Results: Between October 2016 and December 2017, 1,173 patients were admitted to the units with 120 requisitions of target antimicrobials. Sepsis $(43 / 120 ; 35.8 \%)$ was the most common indication, followed by respiratory infections $(23 / 120 ; 19.2 \%)$ and infections in two or more sites $(11 / 120 ; 9.2 \%)$. The most common target antimicrobials requested were meropenem (68/120; 56.7\%), amphotericin B lipid formulations (12/120; 10\%), teicoplanin (11/120; 9.2\%), and linezolid (11/120; 9.2\%). In 98 (81.7\%) cases, previous cultures before antimicrobial administration were collected. An infectious agent was detected in 52 of the 98 cultures (53.1\%), and Gram-negative and Gram-positive bacteria represented $50 \%$ and $26.9 \%$ of all positive samples, respectively. Besides, 111 (92.5\%) requisitions were approved. In five refused requisitions, a narrow-spectrum antimicrobial was prescribed after further discussion; four were refused due to lack of information, and in one case, the de-escalation of the antimicrobial was possible. No mistake regarding dosage and duration was detected.
\end{abstract}

Conclusions: We found a high-quality rate of target antimicrobial requisition. Antimicrobial policy restriction could contribute to improving the quality of antimicrobial prescription, even in NICUs and PICUs.

Keywords: Quality, Antimicrobial Stewardship, Children, Neonates, Intervention

\section{Background}

Antimicrobial stewardship programs (ASPs) have been developed in recent years to improve the correct indication of prescribed antimicrobials and avoid resistance in all healthcare settings, including hospitals, as well as outpatients and patients from long-term care institutions (13). These coordinated actions could be different according to healthcare units, but usually, the core components include audit and feedback of antimicrobials, pre-approval of the target or selected antimicrobials, de-escalation according to culture results, administrative support, multidisciplinary teams, and education of healthcare $\operatorname{staff}(4,5)$. Considering that infections of multi-resistant bacteria are also present in neonatal intensive care units (NICUs) and pediatric intensive care units (PICUs), the control of bacte- rial resistance is a global priority for the World Health Organization (WHO). Limited options of new drugs are available for children, so ASPs are interesting tools to promote the judicious use of antimicrobials $(6,7)$.

Interventions of ASPs have led to successful results in overall antimicrobial consumption reduction, as well as target/broad-spectrum antibiotic reduction, even when neonatal and pediatric critical care units were analyzed $(8,9)$. Analysis of antimicrobial use in healthcare institutions is necessary within the context of ASPs to understand the consumption patterns and establish reduction priorities by defining target antimicrobials according to local/regional profile (10). Days of therapy(DOT) or DOT per 1000 patient-days are common quantitative measures described to evaluate the amount of antimicrobial use in the pediatric population (11). 
One important challenge for ASP is to evaluate the quality of antimicrobial prescriptions. From this point of view, appropriate indication means a correct prescription for a correct infectious disease syndrome, including accurate dosage and duration and avoiding broad-spectrum antimicrobials whenever possible, without damage to patient treatment (12). Most studies are focused on the detection of inappropriate use of antibiotics, with few successful interventions available to improve the quality of prescriptions in NICUs and PICUs $(13,14)$.

\section{Objectives}

This study aimed to analyze the quality of target antimicrobial requisitions after an antimicrobial policy restriction program for children.

\section{Methods}

\subsection{Study Design and Setting}

We performed an observational study in a NICU and three PICUs in Rio de Janeiro city, Brazil. The services are located inside a 135-bed pediatric hospital (ProntobabyHospital da Criança).

The NICU is an 11-bed unit that receives neonates from other hospitals and its own emergency service. The hospital studied does not have a delivery room or obstetric service. The PICU is a 34-bed unit subdivided into three other units according to patient profiling: PICU 1 with 10bed capacity that receives acute critically ill patients; PICU 2 with 15-bed capacity that receives acute critically ill children, but for a short stay; and PICU 3 with 9-bed capacity that receives critically ill patients for long stay (usually with chronic diseases) or infectious diseases that necessitate isolation. All sub-units receive clinical and surgical patients referred from other hospitals or emergency services.

\subsection{Antimicrobial Stewardship Program}

Throughout 2016, several components of an ASP were implemented in the whole hospital, including the ICUs: Assessing the point-prevalence of antimicrobial use every three months, updating antimicrobial guidelines available, using new technologies in microbiological laboratories to accelerate the results of cultures, and more training of healthcare workers regarding the better use of antimicrobials. All ASP components were extensively discussed with healthcare teams, including administrative staff, before their full implementation.

Since 2006, the hospital's infection control committee has actively performed healthcare-associated infection
(HAI) surveillance and discussed cases related to antimicrobial use indication when requested. However, until 2016, there were neither formal hospital policies to analyze the amount and quality of antimicrobial prescribed nor training applied to clinicians on this subject.

In October 2016, an antimicrobial policy restriction was formalized for 14 drugs selected by the infection control committee according to the bacterial resistance profile of the hospital and pricing, to be prescribed only after the pre-approval of a pediatric infectious disease specialist. The antimicrobials selected were amphotericin B lipid formulations, caspofungin, ceftobiprole, colistin (inhaled), daptomycin, ertapenem, imipenem, linezolid, meropenem, micafungin, polymyxin $\mathrm{B}$, teicoplanin, tigecycline, and voriconazole. The flow of target antimicrobial requisition is shown in the supplementary material. In the same month, point-prevalence analysis of antimicrobial consumption was replaced by days of therapy (DOT) measurement to analyze the amounts of all antimicrobials consumed in the critical care units.

\subsection{Quality Analysis of Target Antimicrobial Requisition}

All requisitions regarding target antimicrobials between October 2016 and December 2017 were reviewed. We analyzed the following variables: Indication, type of antimicrobial, dose, duration, collection of cultures before administration, and the agreement of a pediatric infectious disease specialist. There were no exclusion criteria for the analysis of requisitions.

\subsection{Data Analysis}

We did a descriptive analysis of variables using Excel spreadsheet $\circledast$ version 2016 (Microsoft Corp., Redmond, WA, USA). We rated the agreement of a pediatric infectious disease specialist with target antimicrobial requisitions as excellent ( $90-100 \%$ of agreement), $\operatorname{good}(75-89 \%$ of agreement), moderate ( $50-74 \%$ of agreement), and poor $(<50 \%$ of agreement).

\subsection{Ethical Aspects}

The study was approved by the local Ethics Committee (Register 2.386.987 from 19 November 2017).

\section{Results}

Between October 2016 and December 2017, 1,173 patients were admitted to the units, including 324 in the NICU and 849 in the PICUs. During this period, there were 164 requisitions for target-antimicrobials from the whole hospital, including 120 (73.1\%) from the NICU and PICUs. Of the 14 target-antimicrobials, six of them were 
not requested: ceftobiprole, daptomycin, ertapenem, imipenem, micafungin, and tigecycline. Meropenem represented 56.7\%, and amphotericin B lipid formulations as $10 \%$ of all requisitions. Figure 1 presents the frequency of each target antimicrobial requested.

Sepsis was the most common indication for target antimicrobials corresponding to $34.1 \%$ of all solicitations, followed by respiratory infections at $19.2 \%$, and infections in two or more sites at $9.2 \%$. The frequency of all indications is presented in Figure 2.

In 98 of 120 (81.6\%) requisitions, cultures were collected before antimicrobial administration. In 54 requisitions with cultures, the most common sites of collection were tracheal aspirate $(14 / 98 ; 14.2 \%)$, blood $(14 / 98 ; 14.2 \%)$, urine (11/98; $11.2 \%)$, cerebrospinal fluid (5/98; 5.1\%), catheter tip (5/98; 5.1\%), oropharyngeal swab $(1 / 98 ; 1 \%)$, pleural fluid $(1 / 98 ; 1 \%)$, surgical wound $(1 / 98 ; 1 \%)$, bone marrow aspirate $(1 / 98 ; 1 \%)$, and urethral secretion $(1 / 98 ; 1 \%)$. In 44 cases, the site of culture collection was not reported.

On nine occasions, no cultures were collected; in eight patients, this information was not available, and in five cases, cultures were not necessary due to treatment continuation, antimicrobial prophylaxis, or lack of venous access. Considering all patients with cultures collected and patients for whom cultures were not necessary, 17 (14.2\%) patients missed an opportunity to identify an infectious agent in cultures.

An infectious agent was found in 52 of 98 (53.1\%) cultures collected. Gram-negative bacteria represented 50\% of all positive cultures (26/52), followed by Gram-positive as $26.9 \%$ (14/52) and fungi as $23.1 \%$ (12/52). The frequency and microorganisms isolated in cultures are presented in Table 1.

Besides, 111 requisitions were approved by pediatric infectious disease specialists, corresponding to $92.5 \%$ of the total. The rate was considered to be excellent. Of the nine remaining refused requisitions, on five occasions, the case was discussed further, leading to the prescription of a narrow-spectrum antimicrobial; four were refused due to lack of information and in the last one, de-escalation of the antimicrobial was possible. No mistakes regarding dose and duration were found.

\section{Discussion}

Inappropriate antimicrobial prescription represents poor quality of good practice to reduce consumption and help avoid bacterial resistance. Several reports around the world identified a high rate of inappropriate antimicrobial prescription $(15,16)$. The main problems identified are an unnecessary indication, wrong antibiotics chosen,
Table 1. Microorganisms Isolated in Cultures Collected Before Administration of Target Antimicrobials in the Neonatal Intensive Care Unit (NICU) and Pediatric Intensive Care Units (PICUs) $(\mathrm{N}=52)$

\begin{tabular}{|c|c|}
\hline Classes & No. \\
\hline Gram-negative bacteria & $26(50 \%)$ \\
\hline Klebsiella pneumoniae ESBL & 4 \\
\hline Pseudomonas aeruginosa & 4 \\
\hline Pseudomonas aeruginosa $C R$ & 4 \\
\hline Klebsiella pneumoniae & 3 \\
\hline Klebsiella pneumoniae $C R$ & 2 \\
\hline Stenotrophomonas maltophilia & 2 \\
\hline Acinetobacter lwoffii & 2 \\
\hline Enterobacter sp ESBL & 2 \\
\hline Others $^{\text {a }}$ & 3 \\
\hline Gram-positive bacteria & $14(26.9 \%)$ \\
\hline Coagulase-negative staphylococcus & 5 \\
\hline Staphylococcus epidermidis $M R$ & 3 \\
\hline Staphylococcus aureus & 2 \\
\hline Micrococcus & 1 \\
\hline Enterococcus faecalis & 1 \\
\hline MRSA & 1 \\
\hline Streptococcus mitis & 1 \\
\hline Fungi & $12(23.1 \%)$ \\
\hline Candida sp & 8 \\
\hline Candida albicans & 3 \\
\hline
\end{tabular}

Abbreviations: ESBL, Extended-spectrum Beta-lactamase; CR, Carbapenem resistant; MRSA, Methicillin-resistant Staphylococcus aureus.

${ }^{a}$ Serratia liquefaciens (1), Escherichia coli ESBL (1), Burkholderia cepaciae (1)

wrong directions, incorrect posology, prolonged prescriptions, and the use of agents with an excessively broad coverage spectrum (17-19).

In our article, we proposed to analyze the quality of target antimicrobial requisitions after the full implementation of an ASP in the NICU and PICUs where empiric and broad-spectrum antimicrobials are usually necessary to use, due to the high risk of death as a consequence of serious infections. The reduction of antimicrobial consumption with a high rate of quality in prescriptions is possible and reported in the NICUs (20).

To achieve the best possible impact of our ASP, an extensive preparatory phase was conducted before its full implementation, where all healthcare workers involved in the process presented their workflow and contributed to the program with valuable suggestions to work with little interference with daily practice. The engagement of clinicians is considered to be vital for actions related to the im- 


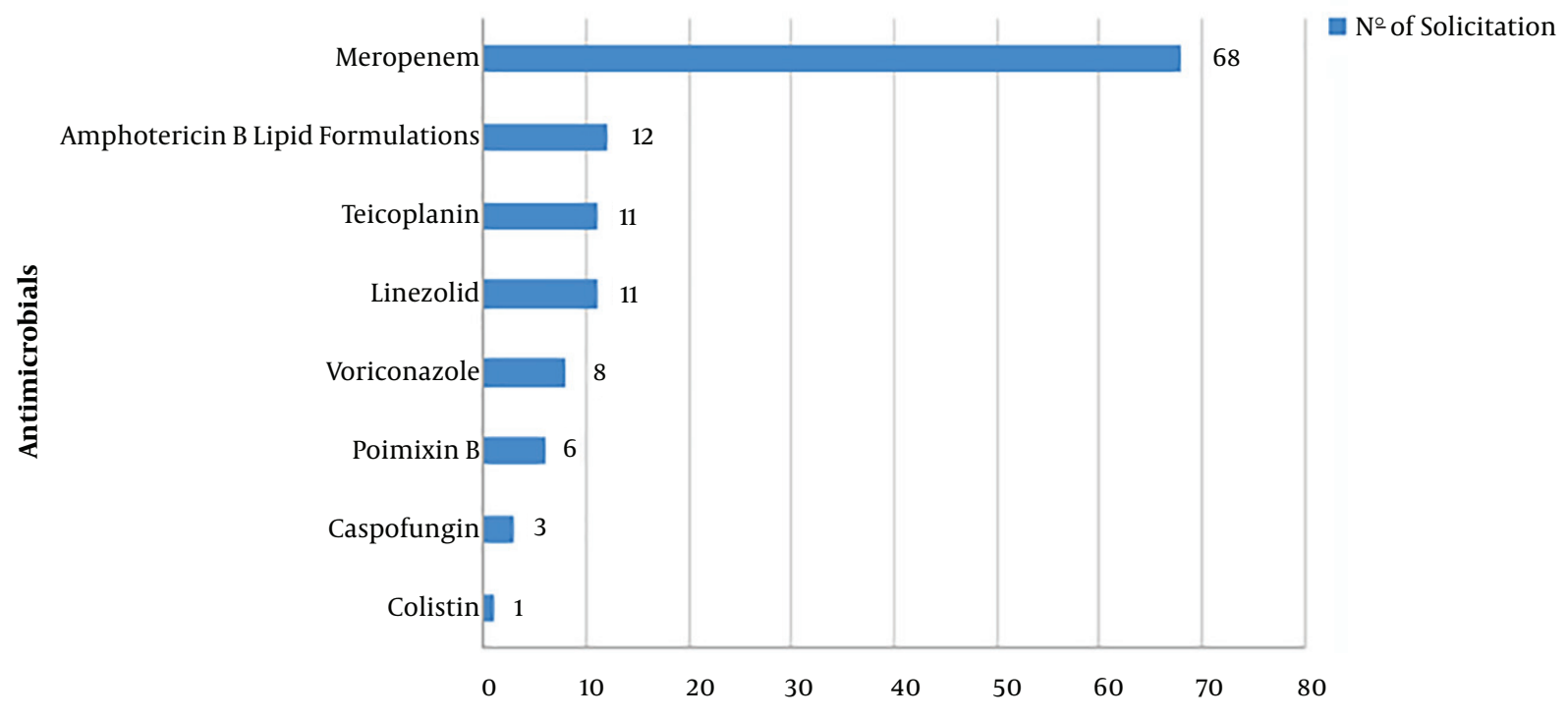

Figure 1. Target Antimicrobial Requisitions in the Neonatal Intensive Care Unit (NICU) and Pediatric Intensive Care Units (PICUs), $\mathrm{N}=120$.

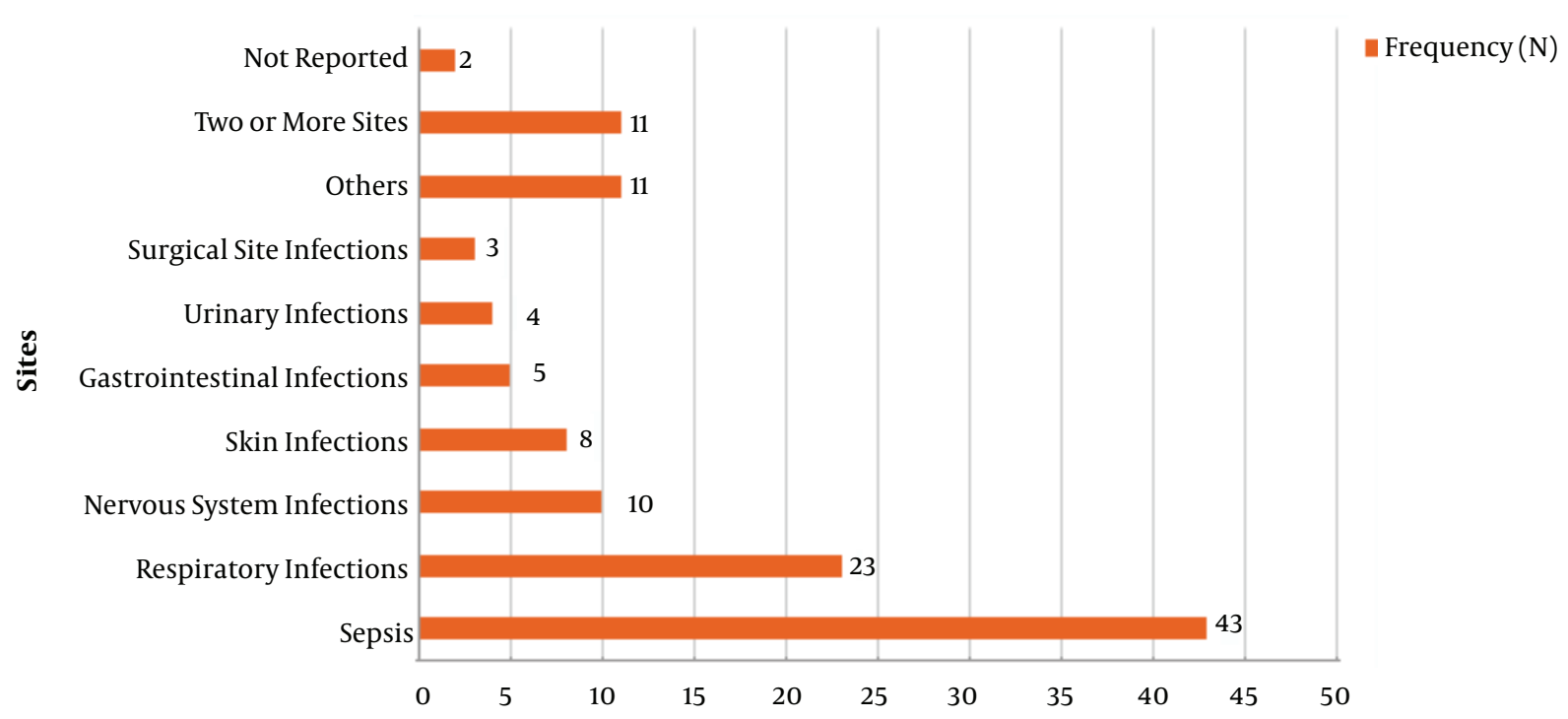

Others: bacterial endocarditis $(n=3)$; prophylaxis $(n=31)$; lack of intravenous access $(n=1)$; febrile neutropenia $(n-I)$; prolonged fever without clinical improvement $(n=1)$; treatment continuation $(n=1)$.

Figure 2. Reasons for target antimicrobial requisitions in the Neonatal Intensive Care Unit (NICU) and Pediatric Intensive Care Units (PICUs).

provement of antibiotic use in hospitals (12).

Sepsis and respiratory infections were the most common reasons for the requisition of target antimicrobials. This finding agrees with previous studies that described indications for antimicrobial use in neonates and children (21). In a point-prevalence study of antimicrobial use con- ducted in 226 hospitals from 41 countries, the most common reason for treating children was bacterial lower respiratory tract infection (18.7\%), and sepsis was the main reason for treating neonates (36.4\%) (21). Sepsis was also the most common reason for an indication for antimicrobial therapy in a long-term series in Sweden (22). 
Gram-negative bacteria, especially Extended-spectrum Beta-lactamase (ESBL) producers, represented important agents of healthcare-associated infections in the hospital studied, and for this reason, empiric treatments, including meropenem, were necessary. Carbapenems are a broadspectrum antibiotic class used for critically ill patients admitted to intensive care units with infections due to ESBL producers, but their indiscriminate use could increase resistance, leading to untreatable infections due to lack of treatment options (23). To preserve all carbapenem activities in our institution, this class was included to be used in the whole hospital, only after discussion and the agreement of pediatric infectious disease specialists. Restricted antimicrobial lists frequently include carbapenems as a class to be used in selected infections due to multidrugresistant bacteria, included as part of a national plan for antibiotic restriction (24-26).

Our study found a high positive culture rate (53.1\%). Although several studies present a blood culture positivity rate around $10 \%,(27)$ it is possible to highlight two factors that probably contributed to this result: Cultures were collected from diverse sites, not only blood; and beyond that, most were collected from critical patients for whom were needed broad-spectrum antimicrobials.

Despite the high rate of culture collections in patients that required target antimicrobials, at least $14 \%$ of them missed the possibility of an infectious agent identification. These "lost opportunities" are avoidable, for example, with the de-escalation of broad-spectrum antimicrobials in favor of narrow-spectrum drugs with the same effectiveness but with less possibility of resistance induction.

In terms of pediatric infectious disease specialist agreement with target antimicrobial requisitions, the rate was considered excellent, reaching more than 92\%, which demonstrates the high capacity of clinicians for identifying correctly the indications for a broad-spectrum antibiotic, including important aspects involved in the quality of prescriptions, such as dose and duration. Our rate was similar to that reported by Luthander and Cols, who found 98.5\% (273/277) of appropriateness in antimicrobial use during a 2003 - 2010 survey in a Swedish pediatric hospital, despite that this report analyzed all hospital wards, not only ICUs.22 Quality programs also contribute to the improvement rate of compliance with guideline recommendation/correction antibiotic administration for selected infectious disease syndromes, such as communityacquired pneumonia or critically ill children assisted in emergency departments $(28,29)$.

This report has some limitations. First, it was conducted in a single-center where it was possible to control factors that interfered with the higher compliance of clinicians to correct indication of target antimicrobials. We be- lieve that more studies using a similar approach involving multiple healthcare institutions could confirm our positive results. Another limitation was the short observation period in which the compliance of clinicians was considered excellent. Further reports should confirm the sustained high quality of target/broad-spectrum antimicrobial prescriptions and ASPs influence on mortality related to infectious diseases over a longer observation period. Finally, previous data about the appropriateness of antimicrobial prescription before intervention were not available to compare the effects in two periods (before and after).

In conclusion, we found a high-quality rate of target antimicrobial requisition. Antimicrobial policy restriction could contribute to improving the quality of antimicrobial prescriptions, even in the NICU and PICUs.

\section{Supplementary Material}

Supplementary material(s) is available here [To read supplementary materials, please refer to the journal website and open PDF/HTML].

\section{Footnotes}

Authors' Contribution: Study concept and design: D.C.A.A.D. and A.R.A.S.; Acquisition of data: D.C.A.A.D. and A.R.A.S.; Analysis and interpretation of data: D.C.A.A.D.; Drafting of the manuscript: D.C.A.A.D.; Critical revision of the manuscript for important intellectual content: A.R.A.S; Statistical analysis: D.C.A.A.D.; Study supervision: D.C.A.A.D. and A.R.A.S.

Conflict of Interests: The authors have no relevant financial interests related to the material in the manuscript.

Ethical Approval: Register 2.386.987 from 19 November of 2017.

Funding/Support: This study had no other sources of funding.

Informed Consent: None.

\section{References}

1. Nathwani D, Varghese D, Stephens J, Ansari W, Martin S, Charbonneau C. Value of hospital antimicrobial stewardship programs [ASPs]: a systematic review. Antimicrob Resist Infect Control. 2019;8:35. doi: 10.1186/s13756-019-0471-0. [PubMed: 30805182]. [PubMed Central: PMC6373132]

2. Drekonja DM, Filice GA, Greer N, Olson A, MacDonald R, Rutks I, et al. Antimicrobial stewardship in outpatient settings: a systematic review. Infect Control Hosp Epidemiol. 2015;36(2):142-52. doi: 10.1017/ice.2014.41. [PubMed: 25632996]. 
3. Katz MJ, Gurses AP, Tamma PD, Cosgrove SE, Miller MA, Jump RLP. Implementing antimicrobial stewardship in long-term care settings: An integrative review using a human factors approach. Clin Infect Dis. 2017;65(11):1943-51. doi: 10.1093/cid/cix566. [PubMed: 29020290]. [PubMed Central: PMC5850640].

4. Barlam TF, Cosgrove SE, Abbo LM, MacDougall C, Schuetz AN Septimus EJ, et al. Implementing an antibiotic stewardship program: Guidelines by the infectious diseases society of America and the society for healthcare epidemiology of America. Clin Infect Dis. 2016;62(10):e51-77. doi: 10.1093/cid/ciw118. [PubMed: 27080992]. [PubMed Central: PMC5006285].

5. Mendelson M, Morris AM, Thursky K, Pulcini C. How to start an antimicrobial stewardship programme in a hospital. Clin Microbiol Infect. 2020;26(4):447-53. doi: 10.1016/j.cmi.2019.08.007. [PubMed: 31445209].

6. El-Nawawy A, Ashraf GA, Antonios MAM, Meheissen MA, El-Alfy MMR. Incidence of multidrug-resistant organism among children admitted to pediatric intensive care unit in a developing country. Microb Drug Resist. 2018;24(8):1198-206. doi:10.1089/mdr.2017.0414. [PubMed: 29589993].

7. WHO. WHO publishes list of bacteria for which new antibiotics are urgently needed. Geneva: World Health Organization; 2017, [cited 1 Sep]. Available from: https://www.who.int/news/item/27-02-2017who-publishes-list-of-bacteria-for-which-new-antibiotics-areurgently-needed.

8. Nzegwu NI, Rychalsky MR, Nallu LA, Song X, Deng Y, Natusch AM, et al. Implementation of an antimicrobial stewardship program in a neonatal intensive care unit. Infect Control Hosp Epidemiol. 2017;38(10):1137-43. doi: 10.1017/ice.2017.151. [PubMed: 28745260].

9. Lee KR, Bagga B, Arnold SR. Reduction of broad-spectrum antimicrobial use in a tertiary children's hospital post antimicrobial stewardship program guideline implementation. Pediatr Crit Care Med. 2016;17(3):187-93. doi: 10.1097/PCC.0000000000000615. [PubMed: 26669645].

10. Urzua S, Ferres M, Garcia P, Sanchez A, Luco M. [Strategies to reduce infections and antibiotic use and its effects in a neonatal care unit]. Rev Chilena Infectol. 2017;34(2):99-107. doi: 10.4067/S071610182017000200001. [PubMed: 28632822].

11. Brotherton AL. Metrics of antimicrobial stewardship programs. Med Clin North Am. 2018;102(5):965-76. doi: 10.1016/j.mcna.2018.05.008. [PubMed: 30126585].

12. CDC. The core elements of hospital antibiotic stewardship programs. Atlanta, USA: Centers for Disease Prevention and Control; 2019 , [cited 1 Sep]. Available from: https://www.cdc.gov/antibiotic-use/ core-elements.

13. Poole NM, Shapiro DJ, Fleming-Dutra KE, Hicks LA, Hersh AL, Kronman MP. Antibiotic prescribing for children in united states emergency departments: 2009-2014. Pediatrics. 2019;143(2). doi: 10.1542/peds.2018-1056. [PubMed: 30622156]. [PubMed Central: PMC6581044].

14. Baig MT, Sial AA, Huma A, Ahmed M, Shahid U, Syed N. Irrational antibiotic prescribing practice among children in critical care of tertiary hospitals. Pak J Pharm Sci. 2017;30(4(Suppl.)):1483-9. [PubMed: 29044002].

15. Mihani J, Kellici S. Patterns of antibiotic prescription in children: Tirana, Albania region. Open Access Maced J Med Sci. 2018;6(4):719-22. doi: 10.3889/oamjms.2018.150. [PubMed: 29731947]. [PubMed Central: PMC5927510].

16. Sviestina I, Mozgis D. Antimicrobial usage among hospitalized children in Latvia: a neonatal and pediatric antimicrobial point prevalence survey. Medicina (Kaunas). 2014;50(3):175-81. doi: 10.1016/j.medici.2014.08.005. [PubMed: 25323546].

17. Croche Santander B, Campos Alonso E, Sanchez Carrion A, Mar- cos Fuentes L, Diaz Flores I, Vargas JC, et al. [Appropriateness of antibiotic prescribing in paediatric patients in a hospital emergency department]. An Pediatr (Barc). 2018;88(5):259-65. doi: 10.1016/j.anpedi.2017.06.001. [PubMed: 28711429].

18. Goycochea-Valdivia WA, Moreno-Ramos F, Pano-Pardo JR, AracilSantos FJ, Baquero-Artigao F, Del Rosal-Rabes T, et al. Identifying priorities to improve paediatric in-hospital antimicrobial use by cross-sectional evaluation of prevalence and appropriateness of prescription. Enferm Infecc Microbiol Clin. 2017;35(9):556-62. doi: 10.1016/j.eimc.2017.01.011. [PubMed: 28284685].

19. Mabiala Babela JR, Ollandzobo Ikobo LC, Mbika Cardorelle A, Moyen G [Prescription of antibiotic drugs for children at the Brazzaville University Hospital Center (Congo)]. Med Sante Trop. 2013;23(2):189-92. doi: 10.1684/mst.2013.0173. [PubMed: 23797761].

20. Zingg W, Pfister R, Posfay-Barbe KM, Huttner B, Touveneau S, Pittet D. Secular trends in antibiotic use among neonates: 2001-2008. Pediatr Infect Dis J. 2011;30(5):365-70. doi: 10.1097/INF.0b013e31820243d3. [PubMed: 21099446].

21. Versporten A, Bielicki J, Drapier N, Sharland M, Goossens H; ARPEC project group. The worldwide antibiotic resistance and prescribing in European children (ARPEC) point prevalence survey: developing hospital-quality indicators of antibiotic prescribing for children. J Antimicrob Chemother. 2016;71(4):1106-17. doi: 10.1093/jac/dkv418. [PubMed: 26747104].

22. Luthander J, Bennet R, Nilsson A, Eriksson M. Antimicrobial use in a swedish pediatric hospital: Results from eight point-prevalence surveys over a 15-year period (2003-2017). Pediatr Infect Dis J. 2019;38(9):929-33. doi: 10.1097/INF.0000000000002393. [PubMed: 31220043].

23. Malchione MD, Torres LM, Hartley DM, Koch M, Goodman JL. Carbapenem and colistin resistance in Enterobacteriaceae in Southeast Asia: Review and mapping of emerging and overlapping challenges. Int J Antimicrob Agents. 2019;54(4):381-99. doi: 10.1016/j.ijantimicag.2019.07.019. [PubMed: 31369812].

24. Saez-Llorens X, Castrejon de Wong MM, Castano E, De Suman O, De Moros D, De Atencio I. Impact of an antibiotic restriction policy on hospital expenditures and bacterial susceptibilities: a lesson from a pediatric institution in a developing country. Pediatr Infect Dis J. 2000;19(3):200-6. doi: 10.1097/00006454-200003000-00005. [PubMed: 10749459].

25. Pakyz AL, Oinonen M, Polk RE. Relationship of carbapenem restriction in 22 university teaching hospitals to carbapenem use and carbapenem-resistant Pseudomonas aeruginosa. Antimicrob Agents Chemother. 2009;53(5):1983-6. doi: 10.1128/AAC.01535-08. [PubMed: 19273670]. [PubMed Central: PMC2681502].

26. Altunsoy A, Aypak C, Azap A, Ergonul O, Balik I. The impact of a nationwide antibiotic restriction program on antibiotic usage and resistance against nosocomial pathogens in Turkey. Int J Med Sci. 2011;8(4):339-44. doi: 10.7150/ijms.8.339. [PubMed: 21647326]. [PubMed Central: PMC3107466].

27. Ergul AB, Isik H, Altintop YA, Torun YA. A retrospective evaluation of blood cultures in a pediatric intensive care unit: a three year evaluation. Turk Pediatri Ars. 2017;52(3):154-61. doi: 10.5152/TurkPediatriArs.2017.5451. [PubMed: 29062249]. [PubMed Central: PMC5644582].

28. Ambroggio L, Thomson J, Murtagh Kurowski E, Courter J, Statile A, Graham C, et al. Quality improvement methods increase appropriate antibiotic prescribing for childhood pneumonia. Pediatrics. 2013;131(5):e1623-31. doi: 10.1542/peds.2012-2635. [PubMed: 23589819] [PubMed Central: PMC3639461].

29. Spencer SP, Karsies T. Audit-and-feedback and workflow changes improve emergency department care of critically Ill children. Pediatr Qual Saf. 2019;4(1). e128. doi: 10.1097/pq9.0000000000000128. [PubMed: 30937410]. [PubMed Central: PMC6426493]. 